\title{
ESTUDO DA VIABILIDADE DE PRODUÇÃO DE TIJOLO ECOLÓGICO MODULAR DE SOLO-CIMENTO COM INCORPORAÇÃO DE RESÍDUO DA FABRICAÇÃO DE PAPEL*
}

\author{
Wellington Fernandes Junior ${ }^{1}$ \\ Maria Angélica Oliveira Vellasco ${ }^{2}$ \\ Israel Nelo Nunes ${ }^{3}$ \\ Jonas Alexandre 4 \\ Sergio Rafael Cortes de Oliveira ${ }^{5}$ \\ Sergio Neves Monteiro ${ }^{6}$ \\ Afonso Rangel Garcez de Azevedo ${ }^{7}$
}

Resumo

O uso de blocos de solo-cimento é considerado um método construtivo mais barato do que o convencional, visto que uma das matérias-primas, o solo, é abundante. Soma-se a isto, o fato de não haver uma destinação correta para o resíduo proveniente da fabricação de papel. Visando redução de custo de produção de tijolos de solo-cimento e uma alternativa sustentável para aproveitamento do resíduo, a proposta do presente trabalho é avaliar a incorporação do resíduo da fabricação de papel na mistura solo-cimento para confecção de tijolos modulares prensados com dimensões de $30 \mathrm{~cm} \times 15 \mathrm{~cm} \times 7 \mathrm{~cm}$. Foram testadas diferentes proporções de solo e resíduo e mantendo sempre a mesma massa de cimento. Foram realizados ensaios de absorção de água e resistência à compressão aos 28 dias de cura. Os resultados foram analisados a fim de verificar a viabilidade da incorporação do resíduo nos tijolos em maior quantidade possível, obedecendo as exigências impostas pelas normas regulamentadoras de blocos de solo-cimento. Foi concluído que a incorporação do resíduo, em quantidades adequadas, é viável na produção de tijolos de solo-cimento, reduzindo o custo da produção, além de diminuir os impactos ambientais com o destino correto do resíduo.

Palavras-chave: Solo-cimento; Resíduo; Tijolo Ecológico; Tijolo Modular.

\section{STUDY OF THE FEASIBILITY OF PRODUCTION OF MODULAR ECOLOGICAL BRICK OF SOIL-CEMENT WITH INCORPORATION OF WASTE OF THE MANUFACTURE OF PAPER}

\begin{abstract}
The use of soil-cement bricks is considered a cheaper construction method than conventional, since one of the raw materials, the soil, is abundant. Added to this is the fact that there is not a correct destination for the waste from papermaking. Aiming to reduce the production cost of soil-cement bricks and a sustainable alternative for the use of the waste, the purpose of this work is to evaluate the incorporation of the papermaking residue in the soil-cement mixture for the manufacture of compressed modular bricks with dimensions of $30 \mathrm{~cm} \times 15 \mathrm{~cm} \times 7 \mathrm{~cm}$. Different proportions of soil and residue were tested and the same cement mass was always maintained. Water absorption and compressive strength tests were performed at 28 days of cure. The results were analyzed in order to verify the viability of the incorporation of the residue in the bricks as much as possible, obeying the requirements imposed by the regulation regulations of soil-cement blocks. It was concluded that the incorporation of the waste, in adequate quantities, is feasible in the production of soil-cement bricks, reducing the cost of production, besides reducing the environmental impacts with the correct destination of the waste.
\end{abstract}

Keywords: Soil-cement; Waste; Ecolological Brick; Modular Brick. 
1 Engenharia Civil, Mestrando em Estruturas, Laboratório de Engenharia Civil, Universidade Estadual do Norte Fluminense (UENF), Campos dos Goytacazes, Rio de Janeiro, Brasil.

2 Graduando em Engenharia Civil, Bolsista de Iniciação Científica, Laboratório de Engenharia Civil, Universidade Estadual do Norte Fluminense (UENF), Campos dos Goytacazes, Rio de Janeiro, Brasil.

3 Graduando em Engenharia Civil, Bolsista de Iniciação Científica, Laboratório de Engenharia Civil, Universidade Estadual do Norte Fluminense (UENF), Campos dos Goytacazes, Rio de Janeiro, Brasil.

4 Engenharia Civil, Doutor, Professor Associado, Laboratório de Engenharia Civil, Universidade Estadual do Norte Fluminense (UENF), Campos dos Goytacazes, Rio de Janeiro, Brasil.

5 Engenharia Civil, Doutor, Professor Titular, Coordenação de Construção Civil, Instituto Federal de Educação, Ciência e Tecnologia Fluminense (IFF), Campos dos Goytacazes, Rio de Janeiro, Brasil.

6 Engenharia Metalúrgica, Doutor, Professor Titular, Laboratório de Ciência dos Materiais, Instituto Militar de Engenharia (IME), Rio de Janeiro, Rio de Janeiro, Brasil.

7 Engenharia Civil, Doutorando em Estruturas, Laboratório de Engenharia Civil, Universidade Estadual do Norte Fluminense (UENF), Campos dos Goytacazes, Rio de Janeiro, Brasil. 


\section{INTRODUÇÃO}

A necessidade da preservação ambiental, associado à escassez dos recursos naturais, fazem com que a construção civil busque por novos conceitos e soluções técnicas visando à sustentabilidade em suas atividades. Para [1], o uso dos tijolos de solo-cimento em uma obra leva a uma redução de custo por volta de $30 \%$, uma vez que o investimento na produção é baixo, facilidade na construção, paredes mais alinhadas, sendo então uma obra simples e com baixo número de profissionais para a construção.

Outro grande problema é o descarte de resíduo solido. A disposição inadequada de resíduos das atividades humanas no ambiente, é um dos temas mais discutidos atualmente [2]. As indústrias de papel e celulose, durante o processo de produção geram grande quantidade de resíduos, o que acarreta em gastos com estocagem ou aumentando os impactos ambientais, visto que são destinados a aterros. O aproveitamento desses resíduos como matéria-prima na produção de novas tecnologias para a construção civil representa a minimização dos danos ambientais, além de ser uma alternativa para a redução do déficit habitacional [3].

O tijolo solo-cimento é um material alternativo de baixo custo, obtido pela mistura de solo, cimento e um pouco de água. Há registros que esse tipo de mistura tem sido utilizado na construção civil há mais de dez mil anos [2]. Segundo [4], estes tijolos representam uma alternativa que está sintonia com desenvolvimento sustentável, pois requerem baixo consumo de energia na extração da matéria-prima, dispensam o processo de queima e reduzem a necessidade de transporte, uma vez que os tijolos podem ser produzidos com solo do próprio local da obra.

Visando a redução de custos de materiais na construção, a utilização de resíduos da fabricação de papel tem sido uma alternativa de destinação ecologicamente correta. Os tijolos de solo-cimento incorporados com resíduo da fabricação de papel é um material alternativo para construção civil, de baixo custo, visto valor da tonelada da argila, e o resíduo que tem custo zero. Desta forma, tornase uma importante alternativa de para construção civil, podendo, com maquinários simples, produzir blocos ou tijolos no próprio local e minimizando a degradação do meio ambiente, atendendo ainda as especificações de qualidade impostas pela Norma Brasileira Regulamentadora. 


\section{MATERIAIS E MÉTODOS}

Neste trabalho foram produzidos tijolos compostos por solo, cimento e resíduo da fabricação de papel. Foram preparadas quatro misturas solo-cimento contendo até $15 \%$ em peso de resíduos de grãos. O solo foi parcialmente substituído pelo resíduo. Cada traço (solo: cimento: resíduos) é rotulado da seguinte forma: T1 - 9:1:0 - traço de solo-cimento de referência, T2 - 8,5:1:0,5, T3-8:1:1 e T4 - 7,5:1:1,5. O solo é proveniente do distrito de São Sebastião, localizado no município de Campos dos Goytacazes, no norte do estado do Rio de Janeiro. O cimento utilizado foi do tipo CP-V ARI, adquirido no comércio local. O resíduo é oriundo de uma indústria de fabricação de papel localizada no município de Santo Antônio de Pádua, no noroeste do estado do Rio de Janeiro. O solo e o resíduo passaram por um processo de secagem, onde foram expostos ao sol até que estivessem seco o suficiente para serem triturados. Em seguida passaram por um triturador com regulagem de tamanho dos grãos, em que foi utilizada uma regulagem para que todo o material passasse pela peneira $n^{\circ} 10$, a fim de diminuir o número de vazios que seriam formados caso fosse utilizado material que tenha grãos maiores que 2,0 mm. Foram realizados ensaios de análise granulométrica, limites de Atterberg e Espectroscopia de Raios $X$ no solo e no resíduo. Após moldados, os blocos foram curados em câmara úmida $\left(95 \%\right.$ de umidade a $\left.23^{\circ} \mathrm{C}\right)$ por 28 dias e em seguida foram submetidos aos ensaios de absorção de água e resistência à compressão.

\section{RESULTADOS E DISCUSSÃO}

\subsection{Análise granulométrica}

A Tabela 1 apresenta a análise granulométrica, de acordo com [5] do solo. Foi constatado através do ensaio de granulometria que o solo utilizado é um solo argilosiltoso.

Tabela 1. Granulometria do solo

\begin{tabular}{lcc}
\hline & GRANULOMETRIA \\
\hline PEDREGULHO & FINO & \\
& GROSSA & $0,40 \%$ \\
AREIA & MÉDIA & $4,70 \%$ \\
& FINA & $9,10 \%$ \\
SILTE & & $11,20 \%$ \\
ARGILA & & $25,60 \%$ \\
\hline
\end{tabular}

O resíduo de papel utilizado apresentou-se como um material silte-argiloso. A granulometria do resíduo de papel está representada na Tabela 2. 
Tabela 2. Granulometria do resíduo da fabricação de papel

GRANULOMETRIA

\begin{tabular}{lcc}
\hline AREIA & MÉDIA & $2,80 \%$ \\
SILTE & FINA & $8,40 \%$ \\
ARGILA & & $50,90 \%$ \\
\hline
\end{tabular}

\subsection{Limites de Atterberg}

O resultado obtido no ensaio de limite de liquidez (LL) para o solo utilizado pode ser observado na Tabela 3. O solo analisado atende ao requisito de [6] em relação ao limite de liquidez, sendo inferior ao máximo permitido de $45 \%$ e em relação ao índice de plasticidade, com valor inferior ao máximo permitido, de 18\%.

Tabela 3. Propriedades físicas do solo

\section{LIMITE DE ATTERBERG}

\begin{tabular}{ccc}
\hline LL & LP & IP \\
\hline $41,10 \%$ & $24,10 \%$ & $17,00 \%$ \\
\hline
\end{tabular}

Os resultados obtidos nos ensaios de limite de liquidez (LL), limite de plasticidade (LP) e índice de plasticidade (IP) para o resíduo utilizado podem ser observados na Tabela 4. Nota-se que o resíduo analisado não atende ao requisito de [6] em relação ao limite de liquidez, sendo superior ao máximo permitido de $45 \%$, assim como não atende em relação ao índice de plasticidade, com valor superior ao máximo permitido, de $18 \%$. Porém estas normas são tomadas como parâmetro para o solo a ser misturado ao cimento. Como o resíduo não será utilizado em substituição total ao solo, mas sim em substituição parcial em pequenas parcelas, estes valores não influenciam no resultado, pois ao substituir pequenas quantidades do solo pelo resíduo, as misturas atendem às normas regulamentadoras. 
Tabela 4. Propriedades físicas do resíduo da fabricação de papel

\section{LIMITE DE ATTERBERG}

\begin{tabular}{ccc}
\hline LL & LP & IP \\
\hline $95,90 \%$ & $72,00 \%$ & $23,90 \%$ \\
\hline
\end{tabular}

\subsection{Espectroscopia de raios $\mathrm{X}$}

\section{Espectroscopia de raios $X$ (EDX)}

A Tabela 5 apresenta os resultados da Espectroscopia de raios X (EDX) do solo quanto à sua composição química, onde é possível observar que o solo é constituído predominantemente de sílica, alumina e ferro. O SiO2 e o Al2O3, estão combinados formando a estrutura da caulinita. O Fe2O3 está relacionado com a estruturação e agregação do solo, conferindo-Ihe maior resistência à erosão. Os demais óxidos presentes são possivelmente de impurezas.

Tabela 5. Espectroscopia de raios X (EDX) do solo

\begin{tabular}{ccccccc}
\hline \multicolumn{7}{c}{ ESPECTROSCOPIA DE RAIO X } \\
\hline $\mathrm{SiO}_{2}$ & $\mathrm{Al}_{2} \mathrm{O}_{3}$ & $\mathrm{Fe}_{2} \mathrm{O}_{3}$ & $\mathrm{SO}_{3}$ & $\mathrm{TiO}_{2}$ & $\mathrm{~K}_{2} \mathrm{O}$ & $\mathrm{CaO}$ \\
\hline $48,38 \%$ & $35,26 \%$ & $11,25 \%$ & $1,93 \%$ & $1,33 \%$ & $1,16 \%$ & $0,39 \%$ \\
\hline
\end{tabular}

A Tabela 6 apresenta os resultados Espectroscopia de raios X (EDX) do resíduo da fabricação de papel, onde é possível observar que o resíduo é constituído predominantemente de óxido de cálcio, sílica, alumina e carbono orgânico. O $\mathrm{CaO}$ está associado à calcita. Já o $\mathrm{SiO} 2$ e o Al2O3, estão combinados formando a estrutura da caulinita. $\mathrm{O}$ carbono está associado à presença de celulose. Os demais óxidos presentes, $\mathrm{MgO}$, $\mathrm{TiO} 2$ e Fe2O3, são possivelmente de impurezas presentes no caulim e na calcita. 
Tabela 6. Espectroscopia de raios X (EDX) do resíduo da fabricação de papel

\begin{tabular}{ccccccc}
\hline \multicolumn{7}{c}{ ESPECTROSCOPIA DE RAIO X } \\
\hline $\mathrm{SiO}_{2}$ & $\mathrm{Al}_{2} \mathrm{O}_{3}$ & $\mathrm{Fe}_{2} \mathrm{O}_{3}$ & $\mathrm{SO}_{3}$ & $\mathrm{TiO}_{2}$ & $\mathrm{~K}_{2} \mathrm{O}$ & $\mathrm{CaO}$ \\
\hline $22,53 \%$ & $18,10 \%$ & $37,87 \%$ & $2,05 \%$ & $0,56 \%$ & $0,82 \%$ & $18,12 \%$ \\
\hline
\end{tabular}

\subsection{Resistência à compressão}

Os tijolos foram ensaiados após 28 dias de cura em câmara úmida. A Figura 1 apresenta o resultado do traço base ( $0 \%$ de resíduo) e dos traços com substituição parcial do solo pelo resíduo. O resultado aponta o valor médio da resistência à compressão obtido a partir de ensaio de seis tijolos de cada traço. Nota-se que a substituição do resíduo pelo solo nas proporções de 5\% e 10\% em massa, atendem ao requisito da [7] em relação à resistência à compressão, já que o valor médio de resistência à compressão aos 28 dias de idade dos tijolos nessas proporções foi superior ao mínimo permitido, de 2,0 MPa.

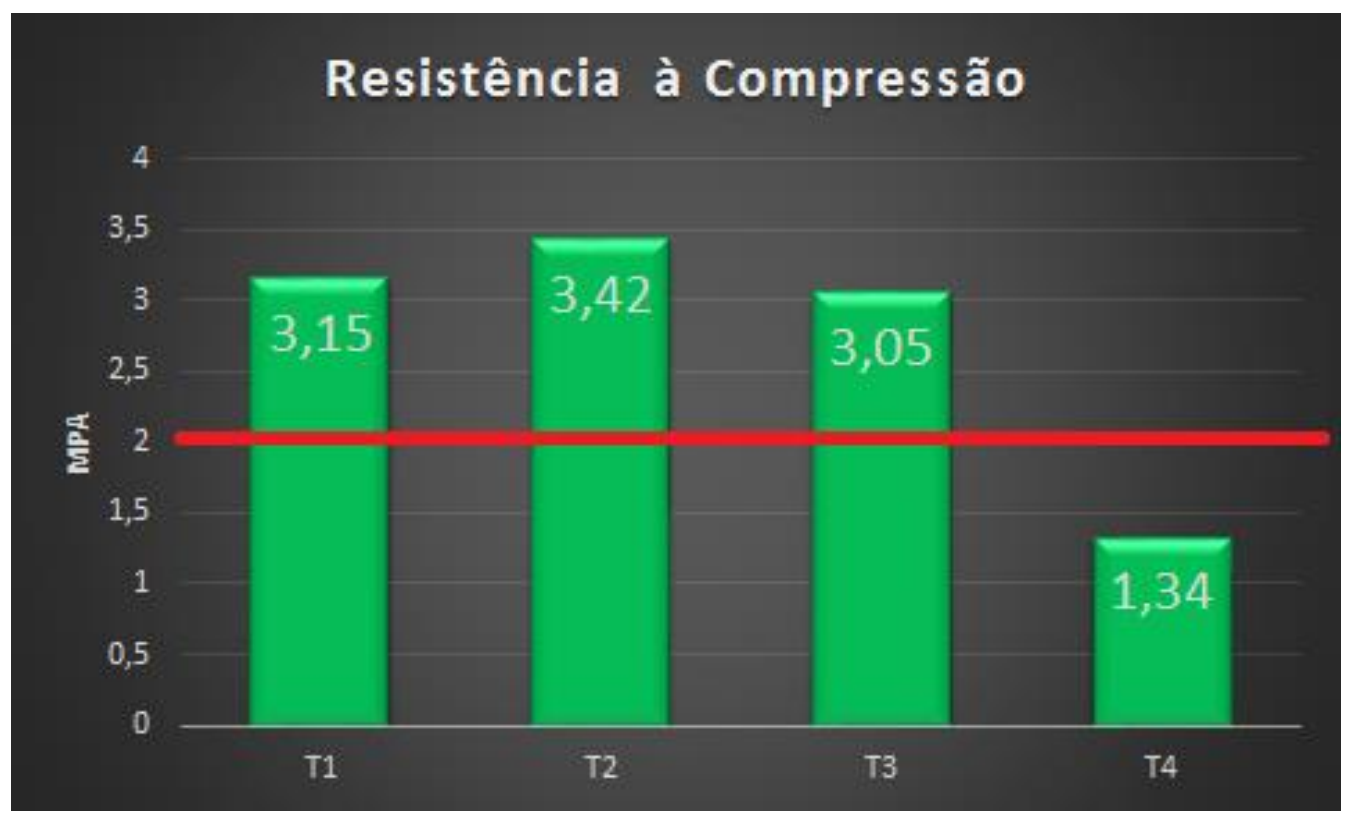

Figura 1. Resistência à compressão média dos tijolos 


\subsection{Absorção de água}

Os tijolos foram ensaiados após 28 dias de cura em câmara úmida. A Figura 2 mostra o resultado do traço base e dos traços com substituição parcial do solo pelo resíduo. O resultado aponta o valor médio da absorção de água obtido a partir de ensaio de seis tijolos de cada traço. Nota-se que a substituição do resíduo pelo solo nas proporções de 5\% e 10\% em massa, atendem ao requisito de [6] em relação à absorção de água, já que o valor médio de absorção de água aos 28 dias de idade dos tijolos nessas proporções foi inferior ao máximo permitido, de $20 \%$.

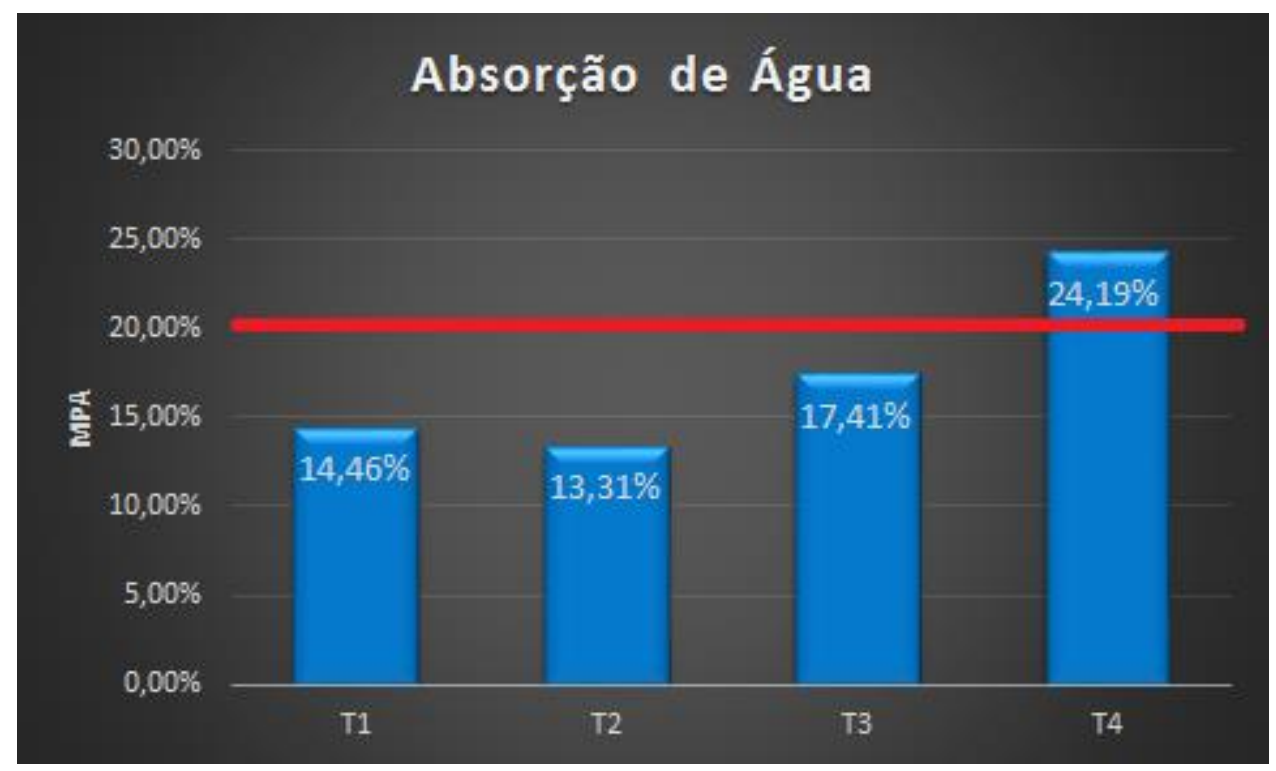

Figura 2. Absorção de água média dos tijolos

\section{CONCLUSÃO}

A fim de verificar a maior porcentagem de resíduo que poderia ser incorporado à massa, atendendo as exigências normativas, foi possível observar que os tijolos com porcentagens de $5 \%$ e $10 \%$ de resíduo atenderam às exigências, sendo definido o teor de $10 \%$ como o teor que utiliza a maior quantidade de resíduo atendendo às exigências normativas.

Portanto, a incorporação do resíduo, em quantidades adequadas, é viável na produção de tijolos modulares de solo-cimento. A presente pesquisa enquadra-se no contexto da sustentabilidade na construção civil pela possibilidade de reciclagem de material, pela dispensa de queima na produção dos tijolos e pela minimização dos 
desperdícios no canteiro de obras associada ao sistema modular, contribuindo, dessa forma, para a diminuição da degradação do meio ambiente e do déficit habitacional.

\section{Agradecimentos}

À FAPERJ pelo suporte financeiro. Os autores também gostariam de agradecer a COMPANHIA PADUANA DE PAPÉIS pelo fornecimento de resíduo da fabricação de papel e a ARTE CERÂMICA SARDINHA pelo fornecimento de solo.

\section{REFERÊNCIAS}

1 PINHEIRO, M. L.; ALVARENGA, R. de C. S.; RIBEIRO, B. C.; SILVA JÚNIOR, P. R.; SARMET, M. S.; FASSONI, D. P. Avaliação experimental de blocos prensados de solocimento com adição de grits. Ambiente Construído, Porto Alegre, v. 13, n. 2, p. 29-46, abr./jun. 2013.

2 ASSOCIACAO BRASILEIRA DE CELULOSE E PAPEL. Relatório Estatístico Mensal. São Paulo: BRACELPA, março de 2011

3 RODRIGUES, S.M. Adição de resíduo de argamassas mistas na produção de tijolos modulares de solo-cimento. 106p. Dissertação (Mestrado em Engenharia do Meio Ambiente, Recursos Hídricos e Saneamento Ambiental). Universidade Federal de Goiás, Goiás, 2008.

4 MONTE, M. A. V. Aplicação de Solo-cimento em Pavimentos Flexíveis. 101p. Dissertação (Mestrado em Engenharia Civil) - Universidade do Porto, Porto, Portugal, 2012.

5 ASSOCIAÇÃO BRASILEIRA DE NORMAS TÉCNICAS. NBR 7181: Solo: Análise granulométrica. Rio de Janeiro, 1984

6 ASSOCIAÇÃO BRASILEIRA DE NORMAS TÉCNICAS. NBR 10832: fabricação de tijolo maciço de solo-cimento com utilização de prensa manual. Rio de Janeiro, 1989..

7 ASSOCIAÇÃO BRASILEIRA DE NORMAS TÉCNICAS. NBR 10836: Bloco vazado de solo-cimento sem função estrutural: Determinação da resistência à compressão e da absorção de água. Rio de Janeiro, 1994. 